Roche's RG7604, which inhibits PI3Ka, PI3K $\delta$ and $\mathrm{PI} 3 \mathrm{~K} \gamma$, is in the adaptive Phase II/III Lung-MAP trial for non-small-cell lung cancer. Several single-isoform inhibitors are also in the clinic.

However, with few exceptions, the "emerging clinical data show limited single-agent activity of inhibitors targeting PI3K" wrote Amgen's Christian Rommel and his co-author in a recent review of the field (Nature Rev. Drug Discov. 13, 140-156; 2014). Better biomarkers and rational combination candidates - such as inhibitors of tyrosine kinases, MEK (mitogen-activated protein kinase kinase), BRAF, MYC, autophagy, poly(ADP-ribose) polymerase, aromatase and $\mathrm{BCL}-2$ - are needed to reinvigorate the class, they write.

\title{
FDA approves second transgenic milk drug
}

Despite regulatory success for Pharming's conestat alfa, 'farmaceuticals' continue to face an uphill battle. The lowdown: The US Food and Drug Administration (FDA) approved Pharming's conestat alfa for the treatment of hereditary angioedema, providing a second complete case study for developers of drugs from transgenic animals. The $\mathrm{C} 1$-esterase inhibitor, which the European Medicines Agency (EMA) approved in 2010, is produced in the milk of genetically modified rabbits and then purified for therapeutic use. Pharming and other transgenic animal drug developers argue that their manufacturing process provides cost-effectiveness and quality benefits over traditional cell-culture production systems. Yet, although BioMedTracker forecasts that annual global sales of the drug will hit US $\$ 52$ million by 2020 , the stated benefits of transgenic-milk products have proven difficult to translate into commercial success.

The only other company to receive an approval for a transgenic-milk drug is rEVO (formerly GTC Biotherapeutics), for ATryn, an anticoagulant antithrombin that is produced in the milk of genetically modified goats. The EMA approved ATryn in 2006 for the prophylaxis of venous thromboembolism for use during surgery on patients with congenital antithrombin deficiency, and this was followed by a similar approval from the FDA in 2009. rEVO has since struggled to profit from the drug. "We have incurred net losses in each year since our inception," wrote rEVO in a recent filing to the US Securities and Exchange Commission. "We expect our expenses will exceed the revenue we collect from sales of ATryn for at least the next several years," they add.

Although many developers of drugs from transgenic animals have fallen by the wayside as the field has fought to establish itself, a few persist. LFB Biotechnologies and its subsidiary rEVO have a few ongoing programmes, including a human factor VIla product from rabbit milk that is in Phase III trials for haemophilia A and haemophilia B. Synageva's sebelipase alfa, a recombinant human lysosomal acid lipase (LAL) that is produced in the eggs of transgenic chickens, is in Phase III trials for early- and late-onset LAL deficiency. 